\title{
Assessment on Nutritional Status of Pregnant Women and Their New Born Baby in the Hospitalized Care System
}

\author{
U. K. Prodhan ${ }^{1}$, M. S. Islam ${ }^{2}$, M. R. Alam ${ }^{1}$, T. K. Vasker ${ }^{1}$, H. Kabir ${ }^{1}$, M. A. Alim ${ }^{3}$, \\ ${ }^{1}$ Department of Food Technology and Nutritional Science, Mawlana Bhashani Science and Technology University (MBSTU), Santosh, \\ Tangail, Bangladesh \\ ${ }^{2}$ Maternal and Child Health Division, International Centre for Diarrhoeal Disease Research, Dhaka, Bangladesh \\ ${ }^{3}$ Department of Food Science and Technology, German University Bangladesh, Gazipur, Bangladesh
}

\section{Email address:}

utpalftns@yahoo.com (U. K. Prodhan), sharifulftns@gmail.com (M. S. Islam), robin08004@gmail.com (M. R. Alam), titash_ftns14@yahoo.com (T. K. Vasker), alim.08017@gmail.com (M. A. Alim)

*Corresponding author

\section{To cite this article:}

U. K. Prodhan, M. S. Islam, M. R. Alam, T. K. Vasker, H. Kabir, M. A. Alim. Assessment on Nutritional Status of Pregnant Women and Their New Born Baby in the Hospitalized Care System. American Journal of Health Research. Vol. 4, No. 6, 2016, pp. $194-198$. doi: 10.11648/j.ajhr.20160406.17

Received: December 1, 2016; Accepted: December 20, 2016; Published: January 18, 2017

\begin{abstract}
The study was conducted to know the nutritional status of pregnant women and their newborn babies $(\mathrm{n}=120)$ at Tangail Medical College and Hospital, Bangladesh. About 16\% illiterate pregnant women were not any nutritional knowledge and delivered their first child before 20 years where, about $89 \%$ surgical delivery was needed and $21 \%$ of newborn did not get colostrum after birth. Although $78 \%$ newborn mothers were practiced exclusive breast feeding whereas, $22 \%$ mothers were given formula milk to their newborn babies. About $23.40 \%$ of the pregnant women checked their health status regularly but $76.6 \%$ women abstain from checkup that play great impact on mother and upcoming child. Moreover, about $55.50 \%$ pregnant women were taken medicine during pregnancy including $83 \%$ of the pregnant women were taken iron tablet whereas, $44.50 \%$ women were not taken medicine during pregnancy. About $83 \%$ of the newborn were weighted $2500 \mathrm{gm}$ and above, whereas the rest of the newborn were weighted from $1500-2500 \mathrm{gm}$. Besides, majority of the respondents were satisfied with hospitalized service and a small number of respondents claimed and unsatisfied with medical and health facilities of the hospital.
\end{abstract}

Keywords: Nutritional Status, Pregnant Women, Newborn Baby, Breast Feeding, Hospitalized Services

\section{Introduction}

Pregnancy is the period of dynamic change for a mother requiring a lot of care because fetus is nourished directly by the mother through placenta, and baby totally relies upon its mother for nutrition, the pregnant woman is to be delivered with an adequate and well-balanced diet [1], to ensure that she attains an satisfactory weight. Correct dietary balance is necessary to ensure sufficient energy intake for adequate growth of fetus without drawing on mother's own tissues to maintain her pregnancy [2]. In pregnancy, good nutrition is crucial to ensure upright maternal health and lessen the risk of birth defects, suboptimal fetal growth and development as well as chronic health problems in their children [3].
Monitoring gestational nutritional status by taking anthropometric measurements is a promising means of enhancing fetal growth [4]. It is found that nutritional intervention focused on woman's health during the reproductive stage, not only in the preconception period but also during the prenatal period, helps achieve adequate newborn nutritional status and is reflected in childhood health and nutritional conditions [5]. However, babies born less than $2500 \mathrm{~g}$ are considered low in birth weight. Most low birth weight in developing countries is due to intrauterine growth retardation which is caused predominately by maternal malnutrition, either before conception or during pregnancy. The effects of malnutrition during childhood or adolescence and during pregnancy probably have an additive 
negative influence on birth weight. Birth weight is crucial to the survival of the infant. It has been found that normal infants in industrial countries have a mortality rate of $2 / 1,000$ while low birth weight infants have a mortality rate of $86 / 1,000$ [6]. If low birth weight babies survive, they have greater rates of morbidity and poorer neurological development (poor vision, decreased educational attainment, and more cerebral palsy, deafness and autism). Impairment to the nervous system upsurges as birth weight falls. Babies with weights greater than $3.5 \mathrm{~kg}$ have 6.8 cases of neurological problems per 1,000 live births compared to babies with weights less than $1.5 \mathrm{~kg}$ who have 200 cases of neurological problems per 1,000 live births [7]. For weight, an indicator used to measure protein-energy status, women in both developing and industrial countries who are heavier before pregnancy deliver heavier babies [8, 9]. Prepregnancy weight can thus be used to predict low birth weight. In a US study, women with a pre-pregnancy weight below $130 \mathrm{lbs}$ or $59 \mathrm{~kg}$ were more than twice as likely to have low birth weight infants when compared to women with pregnancy weights $>130 \mathrm{lbs}$ or $59 \mathrm{~kg}$ [10]. Exclusive breastfeeding were practiced in Bangladesh about 36\% [11] and percentage of malnutrition in children (birth to 59 months) including wasting, stunting, underweight and low birth weight were found 17.4, 43.2, 41 and 36 respectfully [12]. In Bangladesh, Females giving birth by age 20 was found $63 \%$ and children who are exclusively breastfed at ages less than 6 months was found $45 \%$. It was found that the rest of the populations did not follow the proper guidelines of pregnancy and lactation period [13]. Micronutrient deficiencies especially iron and folic acid deficiencies that result in nutritional anemia in children and women and neural tube defects in newborns remain a public health problem in Bangladesh. Poor intake of foods rich in iron and folic acid and multiple infections have resulted in high rates of anemia among pregnant women and children less than two years. Coverage of pre and postnatal iron and folic acid supplements is very low (only $15 \%$ of pregnant women in rural areas take at least 100 tablets during pregnancy) due, in part, to low compliance rates and low coverage of antenatal services. Coverage of multiple micronutrient supplements formulated to address iron and other micronutrient deficiencies is also very low [14]. The aim of this study was to elucidate the authentic and real situation of the nutritional status of pregnant women and their new born baby in case of hospitalized facilities in Bangladesh. It would be supportive for peoples as well as the authority to know the present situation of pregnancy at hospitalized care system in Bangladesh and also useful for policy makers to reform their hospitals.

\section{Methodology}

\subsection{Study Design and Study Period}

The study was a descriptive cross-sectional study, which was focused on nutritional status of pregnant women and newborn in Tangail Medical College and Hospital, Bangladesh. Total duration of the field work for this study was three months of the mid-year of 2015.

\subsection{Sampling Technique and Sample Size}

During study period average fifteen pregnant mothers were came to the hospitals daily and we collected the data from mothers randomly thrice a week by using standard developed questionnaire. Due to time limitation, we collected the information from 120 participants by using Simple random sampling methods from Tangail Medical College and Hospital, Bangladesh.

\subsection{Questionnaire Design and Development}

A standard questionnaire was developed to obtain the relevant information regarding the general information, socio-economic information and individual information. Anthropometric measurement of mothers and their new born baby were also included in the questionnaire. The purpose of the pre-test was to test the content, wording, and expression, the topical sequence of questions and duration of the interview and the reliability of some items. After pre-test, the questionnaires related for quantitative data collection were improved and reformed to ensure content coverage, the reliability and validity of the study. A Questionnaire were developed containing both closed \& open ended Questionnaire to obtain relevant information on anthropometric, socio-economic, dietary \& clinical information. All Questionnaires were presented \& modified as required.

\subsection{Data Collection Procedures}

Individuals wishing to participate in the study were invited to attend the study on a pre-arranged date. All the individuals selected for the study were given an identification number. After received consent from subject, data were collected through a self-reported information questionnaire.

\subsection{Collection of Clinical and Socioeconomic Information}

All of the respondents were interviewed about clinical information. The socioeconomic status such as family size, monthly income, education level of the respondents, part time employment patterns or sources of income were collected. All of the information's were recorded in the respective places of the questionnaire.

\subsection{Anthropometric Measurement}

(1) Body weight: Weight machine was used to measured body weight. The weight was recorded bare footed and the scale was calibrated to zero marking every time before use. The weight was recorded in kilogram.

(2) Height: Height of the study population were measure in standing position with hanging by the side and bare footed, relaxed way, the vertebral column touching the scale. Height was measured to the nearest $0.1 \mathrm{~cm}$. 
(3) BMI: Measurement of Body Mass Index (BMI) as the best method of measuring the nutritional status of prisoners.

$$
\mathrm{BMI}=\frac{\text { Weight in } \mathbf{k g}}{\text { Height } \text { in } \boldsymbol{m}^{2}}
$$

\subsection{Data Collection Tools}

We used weight machine, height scale, pre-coded and open ended questions contained questionnaire and others tools for data collection.

\subsection{Data Management}

Data management included documentation, storage, data editing, and entry and data cleaning prior to data analysis. All the steps were done carefully.

\subsection{Data Analysis}

The data set were first checked, cleaned and entered into the computer from the numerical codes on the form. The data was edited if there is any discrepancy and then cleaned it. The frequency distributions of the entire variables were checked by using SPSS 16 windows program. For tabular, charts and graphical representation Microsoft word and Microsoft excel were used.

\section{Results and Discussion}

The study was conducted on pregnant women and newborn at Tangail Medical College and Hospital to determine the knowledge about the nutritional status of the pregnant women and their newborn babies.

\subsection{Family Income of the Respondents}

The table below indicates the majority of the respondent's family income was between 5000-10000 Takas whereas; only 10 percent of the respondents' family income was more than 15000 Taka.

Table 1. Family income of the respondents.

\begin{tabular}{ll}
\hline Family income (Taka) & Percentage (\%) of respondents \\
\hline $5000-10000$ & 61 \\
$10000-15000$ & 29 \\
$>15000$ & 10 \\
\hline
\end{tabular}

\subsection{Percentage of Age of Mothers}

The figure below shows the percentage of age of pregnant women were $35 \%$ at the age (15-20), 33\% at the age (21-25), $20 \%$ at the age $(26-30)$ and $17 \%$ at the age $(31<)$. But most of the developing and developed countries average age for childbirth was satisfactory than Bangladesh except some African and Least developed countries [15].
Table 2. Percentage of age of mothers.

\begin{tabular}{ll}
\hline Age & Percentage (\%) of the mothers \\
\hline $15-20$ & 35 \\
$21-25$ & 33 \\
$26-30$ & 20 \\
$>30$ & 12 \\
\hline
\end{tabular}

\subsection{Education Level of Newborn Mothers}

Tables 3 shows the newborn's mother education level where under Secondary School Certificate (SSC), above SSC and means of literate was 57, 27 and 16 percentage respectfully.

Table 3. Education level of newborn mothers.

\begin{tabular}{ll}
\hline Education level & Percentage (\%) of the respondents \\
\hline Under SSC & 57 \\
Above SSC & 27 \\
Literate & 16 \\
\hline
\end{tabular}

\subsection{Distribution of Respondent's Regular Checkup, Taken Medicine and Iron Tablet During Pregnant Time}

The pregnant women checkup their body about $23.40 \%$ but $76.6 \%$ women were absent from checkup that could great impact on mother and coming up child. Every pregnant woman needs to visit a doctor regularly. About $83 \%$ pregnant women taken iron tablets whereas, $17 \%$ were not taken it. Although the condition is good if it compared with prevalence of anemia and the prevalence of anemia among pregnant women in Bangladesh is 50 percent, making it a severe public health problem as defined by WHO standards [16]. The graph also shows the $55.50 \%$ pregnant women were taken medicine during pregnancy whereas, $44.50 \%$ women restrain from taking medicine.

Table 4. Information about regular checkup, taken medicine and iron tablet during pregnant time.

\begin{tabular}{llll}
\hline parameter & \multicolumn{4}{l}{ Percentage (\%) of the respondents } \\
\hline & Regular checkup & Taken medicine & Taken iron tablet \\
\hline Yes & 23.40 & 55.50 & 83 \\
No & 76.60 & 44.50 & 17 \\
\hline
\end{tabular}

\subsection{Types of Delivery and Percentage of Childbirth According to Maturity}

The table below shows that $89 \%$ respondents were chosen surgical delivery and the rest were chosen normal delivery. This data is almost opposite to the data found in Statistics from UNICEF State of the World's Children 2009 report and BDHS 2007 (birth delivered at home 85\% and birth delivered with medically assisted person 18\%) [17], although our data was collected from urban hospital that's why it may be opposite from above report which was the scenario of all over the country in Bangladesh. Most of the women need surgery during the delivery due to early marriage and lack of mother physiological maturity. This graph also shows the types of child birth where about $22 \%$ of child birth was premature, whereas majority of the child birth was mature. 
Table 5. Types of delivery and percentage of childbirth according to maturity.

\begin{tabular}{ll}
\hline Parameter & Percentage (\%) of respondents \\
\hline Normal delivery & 11 \\
Surgical delivery & 89 \\
Mature baby & 78 \\
Premature baby & 22 \\
\hline
\end{tabular}

\subsection{Information About Newborn Babies}

The graph below shows the about $83 \%$ of the newborn were weighted $2500 \mathrm{gm}$ and above whereas, only $17 \%$ of the newborn are weighted from 1500-2500gm. This graph also shows that newborn mothers practice exclusive breast feeding is about $78 \%$ and they restrain from formula milk and others. Some mothers give formula milk about $22 \%$ and some mothers give others (honey, water, sugar water etc.).

Table 6. Information about new born babies.

\begin{tabular}{lll}
\hline Parameter & & \% of respondents \\
\hline \multirow{2}{*}{ Weight } & 1500-2500 gram & 17 \\
& Above 2500 & 83 \\
& Exclusive breast feeding & 78 \\
Types of feeding & Formula milk & 2 \\
& Others & 20 \\
\hline
\end{tabular}

\subsection{Distribution of Respondents Mother Weight Gain During Pregnancy}

For respondents who were underweight before becoming pregnant recommended weight gain of between 12.5 and 18 kilograms during pregnancy. For women who had a normal weight before becoming pregnant recommended weight gain of between 11.5 and 16 kilograms during pregnancy. For women who were overweight before becoming pregnant recommended weight gain of between 7 and 11.5 kilograms during pregnancy. For women who were obese before becoming pregnant recommended weight gain of between 5 and 9 kilograms during pregnancy.

\subsection{Information About Colostrum Taken After Childbirth and Hospital Facilities}

Colostrum is important for newborn baby. It is provided by mother after delivery of child. About $89 \%$ infants take mother colostrum after born whereas, $21 \%$ newborn does not take the colostrum due to lack of knowledge about its benefits. As far as, $92 \%$ of the respondents were satisfied with hospital facilities whereas, the rest of the respondents were not satisfied with the hospital service.

\section{Conclusion}

It can be concluded that well-nourished mothers give birth to healthy babies with normal weight $2500 \mathrm{gm}$ or above and malnourished mother deliver under weight babies. So, nutritional status of the mother before pregnancy, during pregnancy and through the lactating period must be kept adequate to get healthy babies. Majority of mothers provide colostrum to their newborn but some mother who does not know about it benefits do not provide the colostrum due to low literacy level and early pregnancy. Most of the mother takes iron tablets and medicine but do not checkup their heath condition regularly. As a result they suffer from different difficulties during pregnancy and delivery time. The respondents are satisfied to the hospital service and choose surgical delivery which discourages the normal delivery. So, we suggest the mothers to follow the proper guidelines during pregnancy as well as their new born babies. Moreover, the authority should know the actual situation and circumstance about hospitalized care system and modify their policy according to the patients' aspiration and we suggest the academicians and researcher to do more research in this sector. Due to time constraint we concentrated only 120 participants and few parameters tested, so a lot of prospect for academicians to find the more facts in this areas.

\section{Acknowledgements}

The authors are grateful to Tangail Medical College and Hospital, Bangladesh and Department of Food Technology and Nutritional Science, Mawlana Bhashani Science and Technology University, Bangladesh for their assistance and support during this work.

\section{References}

[1] Mudambi, R. (1992). Your foods and its Utilization- Nutrition of the Mother and Child, IGNOU: CFN-2: 1-11.

[2] Mridula, D., Mishra, C. P., and Chakravorty, A., (2003): DietaryIntake of Expectant Mother," Indian Journal of Nutrition and Dietetics, 40 (1): 24-30.

[3] Rusescu, A., (2005). Nutritional Status of pregnant women, children under five years old and school children aged six to seven years. Institute for mother and child care, Romania.

[4] Padilha, P. C., Accioly, E., Libera, B. D., Chagas, C., Saunders, C., (2009) Anthropometric assessment of nutritional status in Brazilian pregnant women. Rev Panam Salud Publication. 25 (2): 171-8.

[5] World Health Organization (2006). Promoting optimal fetal development: report of a technical consultation. Geneva.

[6] Wynn, A, M. Crawford, W. Doyle, and S. Wynn (1991). Nutrition of women in anticipation of pregnancy. Nut. \& Health 7: 69-88.

[7] Hackney Hospital (1991). A think-tank on nutrition in the primary prevention of taw birthweight, cerebral palsy and related handicaps. Institute of Brain Chemistry and Human Nutrition, Hackney Hospital.

[8] Nisander, K. and M. Gordon (1972). The women and their pregnancies. DHEW Publication No. (NIH) 73-379. US Department of Health, Education and Welfare. (As reported in Wynn, et al., 1991.) 
[9] Kardjati, S., et al. (1988). Energy supplementation in the last trimester of pregnancy in East Java: I. Effect on birthweight. Br J. Obstet Gynaecol 95:783-794. (As reported in Krasovec and Anderson, 1991.).

[10] Taffel, S. (1980). Maternal weight gain and the outcome of pregnancy. Vital and Health Statistics, U. S. Department of Health and Human Services, Publication (PHS) 86-1922. (As reported in Krasovec and Anderson, 1991.)

[11] Bangladesh Demographic and Health Survey, 2007.

[12] Statistical Yearbook of Bangladesh, Bangladesh Bureau of Statistics, 2004.

[13] Population Reference Bureau- 2002 Women of Our World; 2001 World Population Data Sheet; The World Youth, 2000; and 1999 Breastfeeding Patterns in the Developing World (http://www.worldpop.org/datafinder.htm).

[14] Nutrition Surveillance Programme; Helen Keller International/ IPHN 2006.

[15] First marriage: DHS Program, Women's lives and experiences, 1994. First birth: DHS surveys.

[16] National Institute of Population Research and Training (NIPORT), Mitra and Associates, and ICF International. 2013. Bangladesh Demographic and Health Survey 2011. Dhaka, Bangladesh, and Calverton, MD, USA: NIPORT, Mitra and Associates, and ICF International.

[17] Statistics from UNICEF State of the World's Children 2009 report and BDHS 2007. 